\title{
Carbamazepine induces a bioenergetics disruption to microvascular endothelial cells from the blood-brain barrier
}

Walla Alelwani ${ }^{1}$, Ekramy Elmorsy ${ }^{2,3}$ Shahad W. Kattan ${ }^{4}$, Nouf Abubakr Babteen ${ }^{1}$, Afnan M. Alnajeebi ${ }^{1}$, Ayat Al-Ghafari ${ }^{5}$, and Wayne G. Carter ${ }^{3}$

${ }^{1}$ Department of Biochemistry, College of Science, University of Jeddah, Jeddah 80203, Saudi Arabia.

${ }^{2}$ Department of Forensic Medicine and Clinical Toxicology, Faculty of Medicine, Mansoura University, Mansoura, Egypt.

${ }^{3}$ School of Medicine, University of Nottingham, Royal Derby Hospital Centre, Derby, UK.

${ }^{4}$ Medical Laboratory Department, College of Applied Medical Sciences, Taibah University, Yanbu, Saudi Arabia.

${ }^{5}$ Biochemistry Department, Faculty of Science, King Abdulaziz University, Jeddah, Saudi Arabia.

\begin{abstract}
:
Carbamazepine (CBZ) is a widely employed anti-seizure medication that crosses the bloodbrain barrier (BBB) to exert its anti-convulsant action. The effects of CBZ on components of the BBB have yet to be completely delineated. Hence the current study evaluated the effects of $\mathrm{CBZ}$ upon mitochondrial functionality of BBB-derived microvascular endothelial cells isolated from Albino rats. The influence of $\mathrm{CBZ}$ on cell viability and barrier functions were evaluated by 3-(4,5 dimethylthiazol-2-yl)-2,5-diphenyl-tetrazolium bromide (MTT), lactate dehydrogenase, and electrophysiological assays over a drug concentration range of 0.1-1000 $\mu \mathrm{M}$. Bioenergetics effects were measured via ATP production, mitochondrial complexes I and
\end{abstract}


III activities, lactate production, and oxygen consumption rates (OCRs), and mitochondrial membrane potential, fluidity and lipid content. CBZ was cytotoxic to microvascular endothelial cells in a concentration and duration dependent manner. CBZ significantly diminished the endothelial cell's barrier functions, and impacted upon cellular bioenergetics: reducing mitochondrial complex activities with a parallel decrease in OCRs and increased anaerobic lactate production. CBZ significantly decreased mitochondrial membrane potential and induced an increase of membrane fluidity and decrease in levels of mitochondrial saturated and unsaturated fatty acids. In summary, CBZ disrupted functional activity of BBB endothelial cells via damage and modification of mitochondria functionality at therapeutically relevant concentrations.

Keywords: Blood-brain barrier; Carbamazepine, Microvascular endothelial cells; Mitochondria.

Introduction: Carbamazepine (CBZ) is commonly employed as an anti-seizure drug and treatment for neuropathic pain (Albani et al., 1995; Alrashood, 2016; Gierbolini et al., 2016). It is a first-line medication for partial or generalized tonic and mixed convulsions, used as a treatment for trigeminal neuralgia, and also utilized as a mood stabilizer (Albani et al., 1995; Wiffen et al., 2014). Its mode of action is not completely understood but is primarily related to blockade of pre-synaptic voltage-gated sodium channels to decrease hyperexcitability of neurons (Macdonald and Kelly, 1995; Ambrosio et al., 2002; Sheets et al., 2008; Sills and Rogawski, 2020). CBZ also affects other channels, receptors, as well as signaling pathways such as those downstream of adenylyl cyclase activation (Chen et al., 1996; Ambrosio et al., 2002), but a comprehensive evaluation of CBZ signaling has yet to be undertaken.

CBZ is a dibenzoazepine with structural similarities to tricyclic antidepressants. It is a neutral molecule of molecular mass 236, with high lipophilic character (Beig et al., 2016) (Figure 1); properties well suited for traversing the blood-brain barrier (BBB) to exert its 
central effects. The BBB is composed of endothelial cells that line the vessels as well as other cell types including pericytes and glial cells such as astrocytes that collectively form a protective barrier between the blood and the CNS (Abbott et al., 2010). The unique properties of the microvessels of the CNS include endothelial cells that are mitochondria-rich, and operate without fenestration to limit undesired transport of molecules across the BBB (Yazdani et al., 2019). Furthermore, these cells express surface transporter proteins from the ATP-binding cassette $(\mathrm{ABC})$ transporter superfamily, including P-glycoprotein, that are coupled to ATP usage to drive unidirectional (efflux) movement of endogenous molecules, as well as xenobiotics (Abbott et al., 2010; Daneman, 2012, Yazdani et al., 2019). Therefore maintenance of ATP levels is critical to the performance and integrity of the BBB.

ATP is synthesized by mitochondria via the process of oxidative phosphorylation, and involves the passage of electrons along an electron transport chain (ETC), and coupling of proton transport with ATP synthesis. The ETC is comprised of a series of protein complexes in the inner mitochondrial membrane that transfer electrons to reduce oxygen to water. This electron transport is coupled to directional flow of protons across the mitochondrial membrane, generating a proton motive force that supports ATP synthesis via ATP synthase (complex V) (Kuhlbrant, 2015).

CBZ has a plasma half-life of 30-40 hours, and although widely prescribed, operates with only a relatively narrow therapeutic index. Clinical efficacy is achieved at steady-state blood levels of $4-12 \mu \mathrm{g} / \mathrm{ml}(17-51 \mu \mathrm{M})$, but toxicity is observed at levels above $15-18 \mu \mathrm{g} / \mathrm{ml}$ $(64-76 \mu \mathrm{M})$, is notable at $40 \mu \mathrm{g} / \mathrm{ml}(>170 \mu \mathrm{M})$, with CBZ potentially lethal at $50 \mu \mathrm{g} / \mathrm{ml}(212$ $\mu \mathrm{M})$ (Hojer et al., 1993; Winek et al., 2001). At toxic or overdose levels, CBZ presents with a wide range of symptomology including ataxia, ventricular arhythmias, seizures, respiratory depression, and induction of coma (Spiller et al., 1990; Spiller, 2001). 
Furthermore, even within its therapeutic window, CBZ can exhibit multiple adverse drug effects (ADRs) including hypersensitivity responses; that for some individuals relates to genetic polymorphisms in human leukocyte antigen (HLA) alleles, and differential efficacy that relates to pharmacogenetics of cytochrome P450 enzymes required for phase I and II CBZ metabolism (Albani et al., 1995; Jaramillo et al., 2014; Fricke-Galindo et al., 2018).

Hitherto, a number of the studies have considered the cytotoxicity or genotoxicity of CBZ, although some of these analyses have only focused upon its potential damage as a contaminant to fresh water systems due to its stability and lack of biodegradation (Ferrari et al., 2003, Laville et al., 2004, Li et al., 2010a,b; Han et al., 2018; Fraz et al., 2018,2019; Shao et al., 2019). One mechanism of CBZ cytotoxicity is through induction of reactive oxygen species (ROS) (Li et al. 2010a,b; Shao et al., 2019; Elmorsy E, 2020), although this has not been observed in all cell studies (Laville et al., 2004). However, these studies have not specifically considered an effect of CBZ upon mitochondrial bioenergetics, or assessed the influence of the drug upon barrier properties of endothelial cells. To cover this knowledge gap, we have examined bioenergetic and barrier properties of isolated microvascular endothelial cells from Wistar albino rats after treatment with $\mathrm{CBZ}$ at therapeutic as well as toxic drug concentrations.

\section{Materials:}

\subsection{Chemicals and reagents: Carbamazepine (CBZ) (5H-dibenzo[b,f]azepine-5-} carboxamide), 3-(4,5 dimethylthiazol-2-yl)-2,5-diphenyl-tetrazolium bromide (MTT) and all other chemicals used in this study were purchased from Sigma (St. Louis, MO, USA), unless another source is mentioned. The media components, Ham's F-10 nutrient mixture, fetal bovine serum, and horse serum were obtained from Gibco BRL (Grand Island, NY, USA). CBZ was dissolved in DMSO at a stock concentration of $1 \mathrm{mM}$. Collagenase/dispase and basic fibroblast growth factor (bFGF) were obtained from Roche Molecular Biochemicals (Indianapolis, IN, 
USA). For oxygen consumption rate, modified Hank's was prepared following Daunt et al. (2005) (5.6 mM KCl, $138 \mathrm{mM} \mathrm{NaCl}, 4.2 \mathrm{mM} \mathrm{NaHCO}_{3}, 1.2 \mathrm{mM} \mathrm{NaH}_{2} \mathrm{PO}_{4}, 2.6 \mathrm{mM} \mathrm{CaCl}_{2}, 1.2$ $\mathrm{mM} \mathrm{MgCl} 2,10 \mathrm{mM} \mathrm{Na-HEPES} \mathrm{(pH} \mathrm{7.4),} \mathrm{and} \mathrm{0.1 \%} \mathrm{(w/v)} \mathrm{bovine} \mathrm{serum} \mathrm{albumin} \mathrm{(BSA).}$

2.2. Animals: Wistar Albino rats were used for preparation of microvascular endothelial cells of the BBB. The use of rats for this study was approved by an institutional research board from the Mansoura Faculty of Medicine (Study reference: R2.16/12/18). Rats were sacrificed under anesthesia with sodium pentobarbital.

\section{Methods:}

\subsection{Isolation of rat cerebral microvascular endothelial cells (rCMECs) and CBZ treatments:}

For isolation and culture of rCMECs the method of Calabria et al. (2006) was followed with modifications as described by Liu et al. (2014), and published previously (Elmorsy et al. 2017). For endothelial cell purification, 33\% continuous Percoll gradients were used. Cells were washed and plated on $35 \mathrm{~mm}$ collagen IV/fibronectin-coated plates (both at $0.1 \mathrm{mg} / \mathrm{ml}$ ). For cell culturing, rCMECs were maintained in Endothelial Cell Medium supplemented with $4 \mu \mathrm{g} / \mathrm{ml}$ puromycin and $100 \mathrm{mg} / \mathrm{ml}$ heparin for 3 days. Thereafter, media were prepared without puromycin. For isolated rCMECs characterization, Western blotting was conducted for expression of both P-glycoproteins and breast cancer resistant transporters proteins to confirm expression properties of microvascular endothelial cells, as published previously (Elmorsy et al., 2017) (results not included). CBZ (or vehicle control) was applied directly to cells within culture media. The proportion of CBZ taken up by cells was not quantified.

\subsection{Cytotoxicity assays:}

3.2.1. MTT assays: Isolated cells were seeded at $10^{3}$ cells/well in 96 well plates and cultured until $90 \%$ confluent before treatment with $\mathrm{CBZ}$ at $1,10,100$, and $1000 \mu \mathrm{M}$ concentrations for 
$3,6,12,24$, or 48 hours. Reduction of MTT substrate was quantified spectrophotometrically at $590 \mathrm{~nm}$, according to the manufacturer's protocol. All assay data points were generated from at least 3 separate experiments, with each experiment containing three replicates at each CBZ concentration. Cell viability was quantified relative to vehicle controls set at $100 \%$ viability.

3.2.2. Lactate dehydrogenase ( $\mathbf{L D H}$ ) assays: Cells were prepared as described above for MTT assays and with CBZ treatments of 1-1000 $\mu \mathrm{M}$, for 3-48 hours. LDH assays were conducted using a commercially available kit (Clontech, Mountain View, CA) following the manufacturer's protocol. Wells containing 2\% Triton X-100 were used as a positive control for total cell lysis. The release of LDH in the media was measured spectrophotometrically at 490 nm using a plate reader 'TopCount' (Perkin Elmer, Ueberlingen, Germany). Cytotoxicity was calculated as a percentage LDH release relative to the positive controls. All assay points were performed in triplicate with each experiment containing 3 wells for each CBZ concentration

\subsection{Functional assays:}

The effect of CBZ on rCMECs function was assessed via an examination of CBZ on transcellular transport of Evans Blue, trans-endothelial potential difference (TEPD), and transendothelial electrical resistance (TEER), as described in a previous publications (Elmorsy et al., 2014). Briefly, cells were grown on a semi-permeable membrane in 24 -well inserts (area $0.6 \mathrm{~cm}^{2}$, Millicell, Millipore Corporation). After 72 hours, a stable TEER was established of approximately $22 \Omega \mathrm{cm}^{-2}$ (Supplementary Figure S1), a value consistent with other transwell studies (He et al., 2014; Srinivasan et al., 2015). Cells were then treated with CBZ over the concentration range of $0.1-100 \mu \mathrm{M}$ and for 3-48 hours. TEER and TEPD were measured at the studied time points using a WPI EVOM resistance meter (WPI, UK). To assess endothelial cell transport properties, Evans blue $(0.05 \mathrm{mg} / \mathrm{ml})$ labelled albumin $(4 \%)$ was added to the media in the treated and controls well inserts. The passage of labelled albumin through the endothelial 
cell monolayer was determined by the infranatent by measuring Evan Blue absorbance at 620 nm using a TopCount' device (Perkin Elmer, Ueberlingen, Germany). All data points were performed in triplicate, and data combined from 3 separate experiments.

\subsection{Effect of CBZ on bioenergetics:}

3.4.1. Intracellular ATP measurements: Cells were prepared and treated as described above for MTT assays. The levels of intracellular ATP were then measured based on luminescence of ATP according to a kit protocol (Ab113849, Abcam, Cambridge, UK). ATP levels were quantified relative to control well readings set at $100 \%$. All data points were performed in triplicate, and data combined from 3 separate experiments.

3.4.2. Mitochondrial complex activity measurements: Isolated rCMECs were cultured in 6 well plates $\left(4 \times 10^{3}\right.$ cells/well) and grown until confluent, before treatment with CBZ (0.1-100 $\mu \mathrm{M}, 3-48$ hours). Cells were then homogenized and a mitochondrial-enriched fraction prepared according to Spinazzi et al. (2012). Briefly, cells were trypsinied, washed twice in PBS, centrifuged at $1000 \mathrm{x}$ g for 5 minutes at $4^{\circ} \mathrm{C}$, then cell pellets flash-frozen in liquid nitrogen. After thawing, the pellet was resuspended in $1 \mathrm{~mL}$ of $10 \mathrm{mM}$ ice-cold hypotonic Tris buffer (pH 7.6), and homogenized with a 2-mL glass/teflon tissue grinder. Two hundred $\mu \mathrm{L}$ of sucrose solution $(1.5 \mathrm{M})$ was added, mixed, and the cell homogenate centrifuged at $600 \mathrm{x} \mathrm{g}$ for $10 \mathrm{~min}$ at $2^{\circ} \mathrm{C}$. The supernatant was retained and centrifuged at $14,000 \mathrm{x}$ g for $10 \mathrm{~min}$ at $2^{\circ} \mathrm{C}$. The mitochondrial pellet produced was then resuspended in $0.5 \mathrm{~mL}$ of $10 \mathrm{mM}$ ice-cold hypotonic Tris buffer ( $\mathrm{pH} 7.6)$, flash-frozen in liquid nitrogen, and stored at $-80^{\circ} \mathrm{C}$ until required. Protein concentration was measured using a Bradford assay. The mitochondrial solution was subjected to three cycles of freeze-thawing to disrupt the mitochondrial membranes prior to use. For Complex I measurements, 25-40 $\mu$ g of protein were used per assay, and for Complex III, 10$20 \mu \mathrm{g}$ of protein per assay. Complex I activities were assayed according to the protocol of Janssen et al. (2004). Dichloroindophenol (DCIP) was used as the terminal electron acceptor 
as it is only reduced by Complex I. Complex I activity was measured at $600 \mathrm{~nm}$. Complex III activity was assayed according to the protocol of Spinazzi et al. (2012). Decylubiquinol (100 $\mu \mathrm{M})$ and Cytochrome $\mathrm{c}(75 \mu \mathrm{M})$ were the electron acceptors for Complex III assays, with activity measured at $550 \mathrm{~nm}$. For calibration of mitochondrial complexes activities, rotenone $(10 \mu \mathrm{M})$, and antimycin $\mathrm{A}(10 \mu \mathrm{g} / \mathrm{mL})$ were used as specific inhibitors for Complexes I and III, respectively. Specific complex activity was calculated by comparing the measured fluorescence in the presence and absence of the specific complex inhibitor. Experiments were repeated at least 4 times for each treatment concentration. Specific complex enzyme activity $\left(\mathrm{nmol} \mathrm{min} \mathrm{mg}^{-1} \mathrm{mg}^{-1}\right)=(\Delta$ Absorbance $/ \mathrm{min} \times 1,000) /[($ extinction coefficient $\times$ volume of sample used in $\mathrm{mL}) \times\left(\right.$ sample protein concentration in $\left.\left.\mathrm{mg} \mathrm{mL}^{-1}\right)\right]$. Extinction coefficient for DCIP $=$ $19.1 \mathrm{mM}^{-1} \mathrm{~cm}^{-1}$, extinction coefficient for reduced cytochrome $\mathrm{c}=18.5 \mathrm{mM}^{-1} \mathrm{~cm}^{-1}$ (Spinazzi et al. 2012).

3.4.3. Lactate production assays: Cells were seeded in 24 -well plates at $5 \times 10^{4}$ cells/well, and treated with $\mathrm{CBZ}(0.1-100 \mu \mathrm{M}, 3-48$ hours $)$. Cells were trypsinized, and then spun at $1000 \mathrm{xg}$ for 5 minutes. Pelleted cells were counted whereas the supernatant media was retained and assayed for lactate levels using a lactate assay kit (Biovision, Mountain View, California, USA) according to the manufacturer's instructions. Lactate production was normalized to cell number and expressed as a percentage of control lactate production, as described previously (Al-Ghafari et al., 2019) All data points were performed in triplicate, and data combined from 3 separate experiments. The lactate contents of the controls were considered $100 \%$.

3.4.4. Measurements of oxygen consumption rate: The oxygen consumption rate (OCR) of rCMECs suspensions was measured polarographically using Clark oxygen electrodes (Rank Brothers, Bottisham, UK) by changes in $\mathrm{PO}_{2}$ tension within the chambers, as described by Elmorsy and Smith (2015). Cells were cultured in T25 flasks. Cells were treated with CBZ at 0.1-100 $\mu \mathrm{M}$, but for a single 24 hour incubation before cells were harvested, trypsinized, 
centrifuged, and then resuspended in modified Hank's solution and counted. For OCR assays, basal OCR conditions were measured for 10 minutes prior to the addition of $2 \mu 1$ of $6 \mathrm{mM}$ azide to each chamber $(12 \mu \mathrm{M}$, final concentration). Basal OCR was measured over a 300 -sec period, whereas the azide slope was measured over 60 seconds after azide addition. The $\mathrm{PO}_{2}$ changes were linear in all chambers. All assay data points were was conducted at least 5 times for every treatment concentration, and at 5 studied time points.

3.4.5 Rhodamine-123 assay of mitochondrial membrane potential: Rhodamine-123 (Rh-123) is a lipophilic cationic dye localized to mitochondria depending on the mitochondrial membrane potential (MMP). Mitochondria were prepared fresh and isolated and maintained in a buffer composed of sucrose $(250 \mathrm{mM})$, HEPES $(10 \mathrm{mM}), \mathrm{K}$-EGTA $(100 \mu \mathrm{M}), \mathrm{MgCl}_{2}(2$ $\mathrm{mM}), \mathrm{KH}_{2} \mathrm{PO}_{4}(4 \mathrm{mM}), \mathrm{pH} 7.4$ containing an ADP regenerating system (10 mM glucose and 2.5 U hexokinase). Mitochondria were encased within a cuvette with continuous stirring in a spectrophotometer (F-7000, Hitachi, Chiyoda, Tokyo, Japan) at $25^{\circ} \mathrm{C}$. Rh-123 was added (50 $\mathrm{nM}$ ) and fluorescence monitored (excitation $503 \mathrm{~nm}$, emission $527 \mathrm{~nm}$ ). After 2 minutes CBZ was added to a final concentration of $10 \mu \mathrm{M}$, then increased to $100 \mu \mathrm{M}$ for a further 2 minutes before addition of the mitochondrial inhibitor, antimycin A $(1 \mu \mathrm{g} / \mathrm{ml})$. Experiments were repeated six times for each $\mathrm{CBZ}$ concentration. For each treatment concentration, fluorescence was measured 5 seconds before the next addition.

3.5. Mitochondrial membrane fluidity and fatty acid composition: Cells were seeded in 6well plates at $\left(4 \times 10^{3}\right.$ cells/well), and treated with $\mathrm{CBZ}$ at $1-1000 \mu \mathrm{M}$ for a single time point of 24 hours. Mitochondria were isolated following Spinazzi et al. (2012) for further evaluation of their mitochondrial membrane fluidity (MMF) and fatty acid content. To evaluate the effect of CBZ upon MMF, a trimethylammonium-diphenylhexatriene (TMA-DPH) fluorescent probe was used as described by Waczulikova et al. (2007) with modifications by Pérez-Hernández et al. (2017). For the mitochondrial suspension, $0.5 \mathrm{mg}$ protein/ml were labeled with TMA-DPH 
at a final concentration of $0.25 \mathrm{mM}$. The samples were incubated in the dark for $30 \mathrm{~min}$ with stirring. Then the fluorescence polarization was measured in the same Perkin Elmer Fluorescence Spectrometer with excitation at $365 \mathrm{~nm}$ and emission at $425 \mathrm{~nm}$. The measurements were performed from $10-60^{\circ} \mathrm{C}$ to assess the thermotropic characterization of treated and untreated mitochondrial membranes. Experiments were repeated five times for each concentration

For evaluation of $\mathrm{CBZ}$ on the mitochondrial fatty acid composition, mitochondrial lipids were extracted following Oemer et al. (2018). Trans-esterification was conducted with boron trifluoride (14\% (w/v) in methanol) (Morrison and Smith, 1964). The fatty acid composition of mitochondria was measured by gas chromatography (Hewlett Packard, 6890 series) with a flame ionization detector (FID) using a $30 \mathrm{~m}$. omegawax column (0.25 mm i.d., $0.25 \mathrm{~mm}$ thickness) for separation of fatty acid methyl esters. The used carrier gas was ultrahighly pure nitrogen $(14 \mathrm{ml} / \mathrm{min})$. The temperature was adjusted to $250^{\circ} \mathrm{C}$ for the injector and detector. For the column, the temperature program was adjusted to $180^{\circ} \mathrm{C}$ for 5 minutes with gradual increase in temperature $\left(5^{\circ} \mathrm{C} / \mathrm{min}\right)$ to a final temperature of $240^{\circ} \mathrm{C}$, which was maintained for 18 min. A standard mixture of fatty acid methyl esters was injected for acyl chain identification in mitochondrial samples. Fatty acids composition is reported as mol\%. The unsaturation index (U/S) was obtained from the total sum of unsaturated fatty acids divided by the total sum of saturated fatty acids. Experiments were repeated five times for each concentration.

\subsection{Statistical analysis:}

The concentration of agent producing $50 \%$ inhibition $\left(\mathrm{IC}_{50} \mathrm{~s}\right)$ were calculated from a plot of $\log$ mole concentration (inhibitor or agonist) versus variable response curve, using nonlinear regression curve fitting models with best fit values. For MTT and ATP assays we adjusted 
constraints of the bottom and top of the curve equal to zero and 100, with an assumption that the viability of the vehicle control was $100 \%$, such that inhibition was at $0 \%$. A Hill slope constraint equal to one was used for the fitting of the LDH data. For studying both the concentration and exposure duration effects, a two-way ANOVA was used with Bonferroni post-test for multiple comparisons. For a single comparison between drug treatments and control, a one-way ANOVA with Dunnet's post-test was used. A Kruskall-Wallis one-way ANOVA with Dunn's post-test was performed to consider statistical differences between the saturated/unsaturated fatty acid ratios. All statistical procedures were conducted using PRISM 5 (GraphPad Software Inc., San Diego, CA), with a $p$-value of below 0.05 considered significant.

\section{Results:}

4.1. $C B Z$ was cytotoxic to BBB endothelial cells: $\mathrm{CBZ}$ was cytotoxic to $\mathrm{rCMECs}$ in a concentrations and duration dependent pattern as assessed by MTT, LDH, and ATP assays (Figure 2). Significant cytotoxicity of CBZ was evidenced at concentrations above $1 \mu \mathrm{M}$ after incubation for 3 hours (MTT and ATP assays), and 24 hours (LDH assays) (Supplementary Data, Tables S1-S3). The high sensitivity of ATP levels to therapeutic CBZ concentrations after a 3 hour exposure was mirrored at other time points; with ATP IC 50 concentrations lower that those calculated for MTT assays (Table 1). The half maximal effective concentration $\left(\mathrm{EC}_{50}\right)$ for LDH assays was also calculated (Table 1).

4.2. CBZ altered endothelial cell barrier function: $\mathrm{CBZ}$ exposure to endothelial cells altered their barrier function. CBZ significantly reduced migration of albumin across the monolayer of rCMECs at drug concentrations of $\geq 1 \mu \mathrm{M}$, after incubations of $\geq 6$ hours (Figure $3 \mathrm{~A}$, and Supplementary Tables S4 and S5). These changes correlated with increased ionic conductance (TEDP and TEER) (Figures 3B,C, and Supplementary Tables S4-S6). 
4.3. CBZ reduced mitochondrial functionality: The effect of $\mathrm{CBZ}$ on mitochondrial functions were further investigated through an examination of mitochondrial complex I and complex III activities. CBZ significantly inhibited both mitochondrial complexes I and III as early as 3 hours post-exposure at concentrations of 0.1 and $10 \mu \mathrm{M}$, respectively (Figure 4 and Supplementary Data Table S2 and S3). An exposure of $0.1 \mu \mathrm{M}$ was sufficient to significantly inhibit both mitochondrial complexes after a 24 hour exposure. In parallel, CBZ significantly increased lactate production from a concentration of $0.1 \mu \mathrm{M}$ after a 6 hour exposure (Figure 5A, and Supplementary Data, Tables S2 and S3). Twenty-four hour oxygen consumption rates (OCRs) decreased in a concentrations dependent manner, significantly reduced by $\approx 50 \%$ after an exposure to $10 \mu \mathrm{M}$ CBZ (Figure 5B).

A Rh-123 assay was used to monitor mitochondrial membrane potential (MMP) and this this assessed after incubation with CBZ at exposures of 10 , and $100 \mu \mathrm{M}$. Cellular challenge with CBZ disrupted the MMP with a significant increase of Rh-123 fluorescence by $155 \%$ and $245 \%$ with CBZ concentrations of 10 and $100 \mu \mathrm{M}$, respectively, (Figure 6A,B). Increase fluorescence was also established using the mitochondrial inhibitor, actimycin A (Figure 6A). A TMA-DPH assay was performed to consider the effect of $\mathrm{CBZ}$ on mitochondrial membrane fluidity (MMF), and this showed that CBZ significantly decreased the membrane fluidity of isolated mitochondria after a $24 \mathrm{hr}$ treatment (Figure 6C).

The effect of CBZ on the fatty acid compositions of mitochondria revealed that at high CBZ concentrations $(100$, and $1000 \mu \mathrm{M})$, there was a significant reduction of both saturated (palmitic and stearic fatty acids) and unsaturated fatty acids (palmitoleic, oleic, linoleic, arachidonic, and docosahexaenoic acid), and decrease of the unsaturated/saturated fatty acids ratio (U/S ratio) (Figure 7) relative to vehicle controls.

\section{Discussion:}


This work was conducted to evaluate the toxicity of CBZ to isolated rCMECs. MTT, LDH, and ATP assays demonstrated that CBZ was cytotoxic to these cultured endothelial cells in a concentration and exposure duration dependent manner. From a comparison of the CBZ inhibitor-concentration curves and associated $\mathrm{IC}_{50}$ (Figure 2 and Table 1), it was apparent that MTT metabolism, and moreover, ATP production, was sensitive to inhibition at relatively low CBZ concentrations. The bioreduction of MTT to formazan is primarily attributed to mitochondrial based enzymes and electron carriers, although non-mitochondrial sites of bioreduction have also been localized (Bernas and Dobrucki, 2002). ATP synthesis, via ATP synthase (complex V of the electron transport chain), is also mitochondrial (Kuhlbrant, 2015). Therefore, we hypothesised that the relative reduction of $\mathrm{IC}_{50}$ values for MTT and ATP assays (to those from $\mathrm{LDH}$ assays) was attributed to the damaging effects of $\mathrm{CBZ}$ to mitochondria, and this prompted us to evaluate this mechanism further.

We studied the cytotoxic effects of CBZ over a broad concentration range of $0.1 \mu \mathrm{M}$ to $1 \mathrm{mM}$, concentrations that include previously reported therapeutic, supra-therapeutic, and toxic levels drug levels (Spiller et al., 1990; Hojer et al., 1993; Winek et al., 2001; Spiller, 2001). The benefit of including relatively high drug concentrations is that it can provide an insight to the adverse effects that arise from an overdose within the limited time frame operational for cell culture experiments.

Other studies have also considered the neurotoxicity of CBZ to primary cells; be it rat cerebellar granular cells (Gao and Chang, 1992), rat hippocampal neurons (Ambrósio et al., 2000; Araújo et al., 2004), or rat astrocytes (Pavone and Cardile, 2003). These studies have demonstrated CBZ neurotoxicity, and induction of apoptosis particularly at relatively high exposure concentrations (100 $\mu \mathrm{M}$ or above). One of the mechanisms of CBZ cytotoxicity is via induction of ROS and a reduction of cellular ATP production (Pavone and Cardile, 2003; Araújo et al., 2004; Li et al., 2010a,b; Shao et al., 2019; Elmorsy E, 2020), although this has 
not been observed in all published studies; with CBZ even able to increase ATP levels in neuroblastoma cells (SH-SY5Y) and liver HepG2 cells (Araújo et al., 2004; Laville et al., 2004; Mannerström et al., 2006).

We examined functional (barrier) properties of endothelial cells via the transport of albumin, and this was significantly impaired with CBZ concentrations of $1 \mu \mathrm{M}$ and above. Reduced albumin transport was inversely correlated with increased ionic conductance (TEER) (Supplementary Table S2), as observed previously after treatment of endothelial cells with antipsychotic drugs (Elmorsy et al., 2014). Paracellular transport of albumin could be lowered at high CBZ exposures due to a loss of barrier integrity, but we would expect the CBZ-induced depletion of cellular ATP to also limit ATP-driven albumin transcytosis (Smith and Borchardt, 1989; Poduslo et al. 1994; Hervé et al. 2008; Srinivasan et al., 2015), and thereby contribute to reduced albumin flux. From an in vivo context, at therapeutic concentrations, CBZ may reduce functional integrity of BBB endothelial cells and therefore increase CNS levels of concurrently administered medication, a process that may promote further toxicity and induce ADRs (Albani et al., 1995; Jaramillo et al., 2014; Fricke-Galindo et al., 2018).

We show that CBZ influenced MMP and significantly inhibited both mitochondrial complexes I and III, and at therapeutically relevant concentrations of $10 \mu \mathrm{M}$ after only a 3 hour exposure. We presume these reductions in activities of components of the ETC contributed to reduced ATP synthesis and therefore the depleted cellular ATP levels we observed. This reduced bioenergetic activity was further evidenced by decreased OCRs and increased lactate production via anaerobic metabolism. Furthermore, medication-induced redox damage to mitochondria will likely promote further release of reactive free radicals, and instigate a positive loop of redox stress and cellular damage (Neustadt and Pieczenik 2008; Deavall et al., 2012; Finsterer and Scorza, 2017). Noteworthy, is that the administration of CBZ to epileptic children resulted in reduced ATP production from peripheral leukocytes (Berger et al., 2010), 
and elevation of markers of redox stress when compared to a control group (Tutanc et al., 2015); demonstrative of a CBZ effect on mitochondria and induction of oxidative stress in vivo.

Lastly, we showed that CBZ significantly altered the levels of mitochondrial fatty acids. This may have been caused by inhibition of synthesis, altered lipid remodeling, or due to the vulnerability of fatty acids to lipid peroxidation after the release of toxic free radicals. We report a decrease of the U/S fatty acid ratio at the relatively high CBZ concentrations that typify extensive redox stress. Hence, CBZ-induced damage to mitochondrial bioenergetics may be mediated via reduced MMP and coupling to ATP synthesis, but also through damage and changes to the mitochondrial membrane composition including lipid peroxidation (Schenkel and Bakovic, 2014; Elmorsy 2020).

Consistent with its therapeutic employment, CBZ binds to voltage-gated sodium channels (Yang et al., 2010), and, as a tricyclic imipramine, can also compete with imipramine for binding to the serotonin transporter (Sarker et al., 2010). However, to date, no specific binding sites of CBZ to channels or pores within mitochondria have been identified.

In summary, our study provides an insight into the potential toxicity of CBZ across a concentration range that includes therapeutic as well as toxic concentrations, and specifically considers bioenergetics disruption as a component of the mechanism of toxicity. The ability of CBZ to deplete ATP levels at relatively low concentrations may have particular functional consequences for ATP-driven transport of molecules across the BBB, since these transporters are essential to maintain the integrity of the CNS from hazardous chemicals, drugs, and toxins. It will certainly be interesting to consider in future studies if damage to mitochondria from CBZ links to any of the CBZ ADRs in vulnerable individuals. Furthermore, it will be of importance to investigate if respiratory substrates such as hydroxybutyrate or coenzyme Q that bypass a damaged ETC, or administration of antioxidants (to combat CBZ-induced redox stress), provide useful means to supplement the current use of activated charcoal, or 
extracorporeal therapy such as heamodialysis or plasmapheresis (Pilapil and Petersen 2008; Ghannoum et al., 2014), to treat CBZ intoxications.

\section{References:}

Abbott NJ, Patabendige AA, Dolman DE, Yusof SR, Begley DJ. Structure and function of the blood-brain barrier. Neurobiol Dis. 2010;37(1):13-25.

Albani F, Riva R, Baruzzi A. Carbamazepine clinical pharmacology: a review. Pharmacopsychiatry. 1995;28(6):235-244.

Al-Ghafari A, Elmorsy E, Fikry E, Alrowaili M, Carter WG. The heavy metals lead and cadmium are cytotoxic to human bone osteoblasts via induction of redox stress. PLoS One. 2019;14(11):e0225341.

Alrashood, ST. Carbamazepine in Profiles of drug substances, excipients, and related methodology. Elsevier Academic Press. 2016. 41, 133-321.

Ambrósio AF, Silva AP, Araujo I, Malva JO, Soares-da-Silva P, Carvalho AP, et al. Neurotoxic/neuroprotective profile of carbamazepine, oxcarbazepine and two new putative antiepileptic drugs, BIA 2-093 and BIA 2-024. Eur J Pharmacol. 2000;406(2):191-201.

Ambrósio AF, Soares-Da-Silva P, Carvalho CM, Carvalho AP. Mechanisms of action of carbamazepine and its derivatives, oxcarbazepine, BIA 2-093, and BIA 2-024. Neurochem Res. 2002; 27(1-2): 121-130.

Araujo IM, Ambrosio AF, Leal EC, Verdasca MJ, Malva JO, Soares-da-Silva P, et al. Neurotoxicity induced by antiepileptic drugs in cultured hippocampal neurons: a comparative study between carbamazepine, oxcarbazepine, and two new putative antiepileptic drugs, BIA 2-024 and BIA 2-093. Epilepsia. 2004;45(12):1498-505.

Beig, A., Lindley, D., Miller, J.M., Agbaria, R. and Dahan, A. Hydrotropic solubilization of lipophilic drugs for oral delivery: the effects of urea and nicotinamide on carbamazepine solubility-permeability interplay. 2016. Front Pharmacol. 7, 379.

Berger, I., Segal, I., Shmueli, D. and Saada, A. The effect of antiepileptic drugs on mitochondrial activity: a pilot study. 2010. J child neurol. 25(5), 541-545.

Bernas, T., Dobrucki, J. Mitochondrial and nonmitochondrial reduction of MTT: interaction of MTT with TMRE, JC-1, and NAO mitochondrial fluorescent probes. 2002. Cytometry 47, 236-242. 
Calabria, A.R., Weidenfeller, C., Jones, A.R., De Vries, H.E. and Shusta, E.V. Puromycinpurified rat brain microvascular endothelial cell cultures exhibit improved barrier properties in response to glucocorticoid induction. 2006. J Neurochem. 97(4), 922-933.

Chen G, Pan B, Hawver DB, Wright CB, Potter WZ, Manji HK. Attenuation of cyclic AMP production by carbamazepine. 1996. J Neurochem. 67(5), 2079-2086.

Daneman, R. The blood-brain barrier in health and disease. 2012. Ann neurol. 72(5), 648-672. Daunt, M., Dale, O., Smith, P.A. Somatostatin inhibits oxidative respiration in pancreatic $\beta$ cells. 2006. Endocrinology, 147(3), 1527-1535.

Deavall DG, Martin EA, Horner JM, Roberts R. Drug-induced oxidative stress and toxicity. J Toxicol. 2012;2012:645460.

Elmorsy, E.E. Carbamazepine induces oxidative stress on rats' microvascular endothelial cells of the blood-brain barrier. 2020. Ann Clin Anal Med 11, 231-234.

Elmorsy E, Smith PA. Bioenergetic disruption of human micro-vascular endothelial cells by antipsychotics. Biochem Biophys Res Commun. 2015;460(3):857-62.

Elmorsy E, Elzalabany LM, Elsheikha HM, Smith PA. Adverse effects of antipsychotics on micro-vascular endothelial cells of the human blood-brain barrier. Brain Res. 2014;1583:25568.

Elmorsy E, Al-Ghafari A, Almutairi FM, Aggour AM, Carter WG. Antidepressants are cytotoxic to rat primary blood brain barrier endothelial cells at high therapeutic concentrations. 2017. Toxicol In Vitro. 44, 154-163.

Ferrari, B, Paxeus, N, Giudice, RL, Pollio, A, Garric, J. Ecotoxicological impact of pharmaceuticals found in treated wastewaters: study of carbamazepine, clofibric acid, and diclofenac. 2003. Ecotoxicol Environ Saf. 55(3), 359-370.

Finsterer, J, Scorza, FA. Effects of antiepileptic drugs on mitochondrial functions, morphology, kinetics, biogenesis, and survival. 2017. Epilepsy res. 136, 5-11.

Fraz, S, Lee, AH, Wilson, JY. Gemfibrozil and carbamazepine decrease steroid production in zebrafish testes (Danio rerio). 2018. Aq Toxicol. 198, 1-9.

Fraz, S, Lee, AH, Pollard, S, Srinivasan, K, Vermani, A, David, E, Wilson, JY. Paternal exposure to carbamazepine impacts zebrafish offspring reproduction over multiple generations. 2019. Environ Sci Tech. 53(21), 12734-12743.

Fricke-Galindo I, LLerena A, Jung-Cook H, Lopez-Lopez M. Carbamazepine adverse drug reactions. 2018. Expert Rev Clin Pharmacol.11(7):705-718.

Gao XM, Chuang DM. Carbamazepine-induced neurotoxicity and its prevention by NMDA in cultured cerebellar granule cells. Neurosci Lett. 1992;135(2):159-62. 
Ghannoum M, Yates C, Galvao TF, Sowinski KM, Vo TH, Coogan A, Gosselin S, Lavergne V, Nolin TD, Hoffman RS; EXTRIP workgroup. 2014. Extracorporeal treatment for carbamazepine poisoning: systematic review and recommendations from the EXTRIP workgroup. Clin Toxicol. 52(10), 993-1004.

Gierbolini J, Giarratano M, Benbadis SR. Carbamazepine-related antiepileptic drugs for the treatment of epilepsy - a comparative review. Expert Opin Pharmacother. 2016;17(7):885-8.

Han, Y., Ma, M., Li, N., Hou, R., Huang, C., Oda, Y. and Wang, Z. Chlorination, chloramination and ozonation of carbamazepine enhance cytotoxicity and genotoxicity: Multiendpoint evaluation and identification of its genotoxic transformation products. 2018. J hazard mater. 342, 679-688.

He, Y., Yao, Y., Tsirka, S.E., Cao, Y. Cell-culture models of the blood-brain barrier. 2014. Stroke 45(8), 2514-2526.

Hervé, F., Ghinea, N., Scherrmann, J.-M., 2008. CNS delivery via adsorptive transcytosis.

AAPS J. 10, 455-472.

Hojer J, Malmlund HO, Berg A. Clinical features in 28 consecutive cases of laboratory confirmed massive poisoning with carbamazepine alone. 1993. J Toxicol Clin Toxicol. 31(3):449-458.

Janssen, A.J. Trijbels FJ, Sengers RC, Smeitink JA, van den Heuvel LP, Wintjes LT, Stoltenborg-Hogenkamp BJ, Rodenburg RJ. Spectrophotometric assay for complex I of the respiratory chain in tissue samples and cultured fibroblasts. 2007. Clin Chem. 53(4), 729734.

Jaramillo NM, Galindo IF, Vazquez AO, Cook HJ, A LL, Lopez ML. 2014. Pharmacogenetic potential biomarkers for carbamazepine adverse drug reactions and clinical response. Drug Metabol Drug Interact. 29(2):67-79.

Kuhlbrandt W. Structure and function of mitochondrial membrane protein complexes. BMC Biol. 2015;13:89.

Laville N, Ait-Aissa S, Gomez E, Casellas C, Porcher JM. Effects of human pharmaceuticals on cytotoxicity, EROD activity and ROS production in fish hepatocytes. 2004. Toxicology, 196(1-2), 41-55.

Li, Z.H., Li, P., Rodina, M. and Randak, T. Effect of human pharmaceutical Carbamazepine on the quality parameters and oxidative stress in common carp (Cyprinus carpio L.) spermatozoa. 2010a. Chemosphere. 80(5), 530-4.

Li, Z.H., Zlabek, V., Velisek, J., Grabic, R., Machova, J. and Randak, T. Modulation of antioxidant defence system in brain of rainbow trout (Oncorhynchus mykiss) after chronic 
carbamazepine treatment. 2010b. Comp Biochem Physiol C Toxicol Pharmacol. 151(1), 13741.

Liu, Q., Hou, J., Chen, X., Liu, G., Zhang, D., Sun, H. and Zhang, J. P-glycoprotein mediated efflux limits the transport of the novel anti-Parkinson's disease candidate drug FLZ across the physiological and PD pathological in vitro BBB models. 2014. PLoS One 9(7):e102442.

Macdonald, R.L. and Kelly, K.M. Antiepileptic drug mechanisms of action. 1995. Epilepsia 1995; 36 (suppl);S2-12.

Mannerstrom M, Toimela T, Ylikomi T, Tahti H. The combined use of human neural and liver cell lines and mouse hepatocytes improves the predictability of the neurotoxicity of selected drugs. Toxicol Lett. 2006;165(2):195-202.

Morrison WR, Smith LM. Preparation of Fatty Acid Methyl Esters and Dimethylacetals from Lipids with Boron Fluoride--Methanol. J Lipid Res. 1964;5:600-8.

Neustadt J and Pieczenik SR. Medication-induced mitochondrial damage and disease. Mol Nutr Food Res. 2008; 52(7):780-8.

Oemer G, Lackner K, Muigg K, Krumschnabel G, Watschinger K, Sailer S, et al. Molecular structural diversity of mitochondrial cardiolipins. Proc Natl Acad Sci U S A. 2018;115(16):4158-63.

Pavone A, Cardile V. An in vitro study of new antiepileptic drugs and astrocytes. Epilepsia. 2003;44(s10):34-9.

Perez-Hernandez IH, Dominguez-Fuentes JM, Palomar-Morales M, Zazueta-Mendizabal AC, Baiza-Gutman A, Mejia-Zepeda R. Liver mitochondrial membrane fluidity at early development of diabetes and its correlation with the respiration. J Bioenerg Biomembr. 2017;49(3):231-9.

Pilapil M, Petersen J. 2008. Efficacy of hemodialysis and charcoal hemoperfusion in carbamazepine overdose. Clin Toxicol. 46, 342-343.

Poduslo, J.F., Curran, G.L., Berg, C.T., 1994. Macromolecular permeability across the blood-nerve and blood-brain barriers. PNAS 91, 5705-5709.

Sarker S, Weissensteiner R, Steiner I, Sitte HH, Ecker GF, Freissmuth M, Sucic S. The highaffinity binding site for tricyclic antidepressants resides in the outer vestibule of the serotonin transporter. Mol Pharmacol. 78(6), 1026-1035.

Schenkel LC, Bakovic M. Formation and regulation of mitochondrial membranes. Int J Cell Biol. 2014;2014:709828. 
Shao Y, Chen Z, Hollert H, Zhou S, Deutschmann B, Seiler TB. 2019. Toxicity of 10 organic micropollutants and their mixture: Implications for aquatic risk assessment. Sci Total Environ. $666,1273-1282$.

Sheets PL, Heers C, Stoehr T, Cummins TR. Differential block of sensory neuronal voltagegated sodium channels by lacosamide [(2R)-2-(acetylamino)-N-benzyl-3methoxypropanamide], lidocaine, and carbamazepine. 2008. J Pharmacol Exp Ther 326:8999.

Sills GJ, Rogawski MA. Mechanisms of action of currently used antiseizure drugs. Neuropharmacology. 2020;168:107966.

Smith, K.R., Borchardt, R.T., 1989. Permeability and mechanism of albumin, cationized albumin, and glycosylated albumin transcellular transport across monolayers of cultured bovine brain capillary endothelial cells. Pharm. Res. 6, 466-473.

Spiller, H.A. Management of carbamazepine overdose. 2001. Clin Pediatr Emerg Med. 17(6), $452-456$.

Spiller HA, Krenzelok EP, Cookson E. Carbamazepine overdose: a prospective study of serum levels and toxicity. 1990. J Toxicol Clin Toxicol. 28(4):445-458.

Spinazzi, M., Casarin, A., Pertegato, V., Salviati, L. and Angelini, C. Assessment of mitochondrial respiratory chain enzymatic activities on tissues and cultured cells. 2012. Nat protoc. 7(6), 1235.

Srinivasan B, Kolli AR, Esch MB, Abaci HE, Shuler ML, Hickman JJ. TEER measurement techniques for in vitro barrier model systems. 2015. J Lab Autom 20(2), 107-26.

Tutanc, M., Aras, M., Dokuyucu, R., Altas, M., Zeren, C., Arica, V., Ozturk, O.H., Motor, S. and Yilmaz, C. Oxidative status in epileptic children using carbamazepine. 2015. Iran j Pediatr. $2015 ; 25(6)$.

Waczulikova I, Habodaszova D, Cagalinec M, Ferko M, Ulicna O, Mateasik A, et al. Mitochondrial membrane fluidity, potential, and calcium transients in the myocardium from acute diabetic rats. Can J Physiol Pharmacol. 2007;85(3-4):372-81.

Wiffen PJ, Derry S, Moore RA, Kalso EA. Carbamazepine for chronic neuropathic pain and fibromyalgia in adults. Cochrane Database Syst Rev. 2014;(4):CD005451.

Winek, C.L., Wahba, W.W., Winek Jr, C.L. and Balzer, T.W. Drug and chemical blood-level data 2001. 2001. Forensic Sci Int. 122(2-3), 107-123.

Yang YC, Huang CS, Kuo CC. Lidocaine, carbamazepine, and imipramine have partially overlapping binding sites and additive inhibitory effect on neuronal $\mathrm{Na}+$ channels. 2010. Anesthesiology 2010. 113(1), 160-174. 
Yazdani, S., Jaldin-Fincati, J.R., Pereira, R.V.S., Klip, A. Endothelial cell barriers: Transport of molecules between blood and tissues. 2019. Traffic 20, 390-403.

\section{Figures Legends:}

Fig. 1. Chemical structure of carbamazepine.

Fig. 2. Cytotoxicity assays of CBZ at concentrations of $0.1,1,10$, and $100 \mu \mathrm{M}$ to rCMECs. Cell viability was assessed via reduction of MTT (A), LDH leakage (B), and intracellular ATP levels $(\mathbf{C})$. Data shown represent means \pm SD.

Fig. 3. Functional effects of CBZ at concentrations of $0.1,1,10$, and $100 \mu \mathrm{M}$ on rCMECs barrier properties for 3, 6, 12, 24, and 48 hours. Barrier properties were assessed using an insert model of Evans Blue (EB) labelled albumin migration (A), trans-endothelial potential difference (TEPD) (B), and trans-endothelial electrical resistance (TEER) (C). Data shown represent means \pm SEM. Interactions between variables (as assessed by two-way ANOVA) are included.

Fig. 4. The effect of $\mathrm{CBZ}$ at concentrations of $0.1,1,10$, and $100 \mu \mathrm{M}$ on the mitochondrial complex I (MCI) (A) and complex III (MCIII) (B) activities of rCMECs. Data shown represent means \pm SD. For significant changes from control: "c" $=p<0.0001$. Interactions between variables (as assessed by two-way ANOVA) are included.

Fig. 5. The effect of CBZ at concentrations of $0.1,1,10$, and $100 \mu \mathrm{M}$ and exposures of 3-48 hours on lactate production from rCMECs (A). Oxygen consumption rates (OCR) after a 24 hour CBZ exposure Interactions between variables (as assessed by two-way ANOVA) are included. (B). Data shown represent means \pm SD. For significant changes from control: "a" = $\mathrm{p}<0.05, " \mathrm{c} "=\mathrm{p}<0.0001$. 
Fig. 6. The effect of CBZ at final concentrations of 10 and $100 \mu \mathrm{M}$ on mitochondrial membrane potential (MMP) (A,B). The effect of actimycin A, a mitochondrial inhibitor, upon the MMP was also demonstrated to verify mitochondrial viability (A). The effect of a $24 \mathrm{~h}$ exposure to CBZ on isolated mitochondria membrane fluidity was assessed by a TMA-DPH assay (C). Data shown represent means \pm SD. For significant changes from control: "c" $=p<0.0001$. Interactions between variables (as assessed by two-way ANOVA) are included.

Fig.7. The effect of CBZ at concentrations of $1,10,100$, and $1000 \mu \mathrm{M}$ on the mitochondrial membrane lipid contents of saturated (palmitic and stearic fatty acid) and unsaturated fatty acids (palmitoleic, oleic, linoleic, arachidonic, and docosahexaenoic acid), and the unsaturated/saturated fatty acids ratio (U/S ratio). Data shown represent means \pm SD. For significant changes from control: "a" = p<0.05, "b" = p<0.001, "c" = p<0.0001. 